\title{
PROVAS CRUCIAIS DA DICENTRICIDADE DOS CROMOSSOOMIOS DOS HEMIPTEROS
}

\author{
S. DE TOLEDO PIZA JOR.
}

\author{
Professor de Zoologia, Anatomia e Fisiologia da Escola \\ Superior de Agricultura "Luiz de Queiroz", da \\ Universidade de São Paulo
}

\begin{abstract}
INDICE

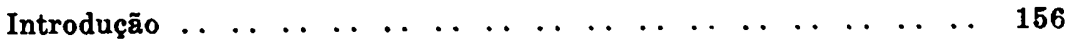

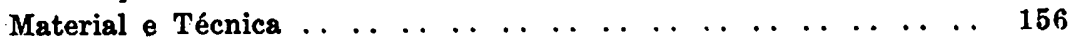

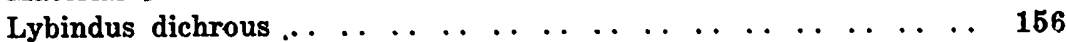

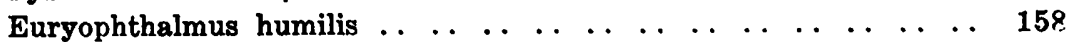

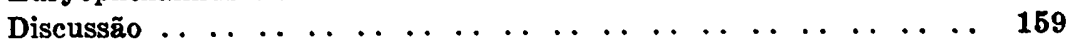

Resumo $\ldots \ldots \ldots \ldots \ldots 165$

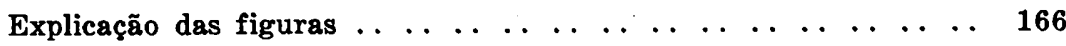

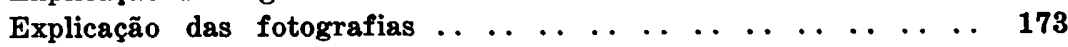

The crucial proofs of the dicentricity of Hemiptera chromo-

somes .. . . . . . . . . . . . . . . . . . . . . . . . . . . 174

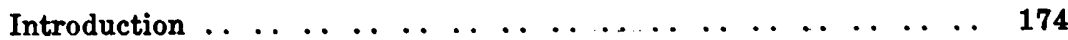

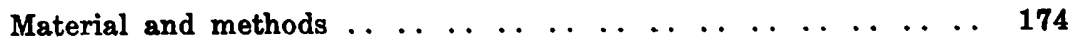

Lybindus dichrous $\ldots \ldots \ldots$

Euryophthalmus humilis $\ldots \ldots \ldots \ldots$

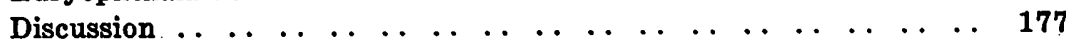

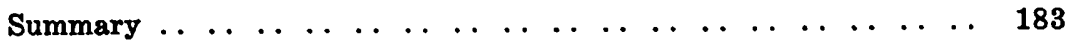

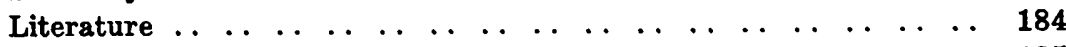

Explanation for the figures . . . . . . . . . . . . . . . . . 185

Explanation for the photographies ... . . . . . . . . . . 186
\end{abstract}




\section{INTRODUÇÃO}

A espermatogênese dos Hemípteros tem sido objeto de um grande número de pesquisas, sendo riquíssima a literatura a respeito. O comportamento particular dos cromossômios dos insetos dessa Ordem levou os autores a um sem número de discussões, havendo ainda sérias dúvidas a respeito de pontos fundamentais. Seguindo SCHRADER (1935), a quase totalidade dos estudantes da citologia dos Hemípteros considera os cromossômios daqueles insetos como sendo providos de um centrômero difuso. Porém, a descoberta de cromossômios possuindo um centrômero em cada extremidade, tal como se constata em escorpiões do gênero Tityus, (PIZA 1939, 1941, 1943a, 1943b), levou o presente autor a reinvestigar a questão e a concluir, que também nos Hemípteros os cromossômios são dicêntricos.

Neste pequeno trabalho novas provas são apresentadas em apôio das conclusões anteriores, uma das quais, oferecida por uma das tétrades de Lybindus dichrous, constitui um dos fatos mais interessantes até agora encontrados na meiose dos Hemípteros.

\section{MATERIAL E TÉCNICA}

O material utilizado no presente estudo consistiu de cêrca de 20 machos do Coreídeo Lybindus dichrous Stal e um único exemplar, do mesmo sexo, do Pirrocorídeo Euryophthalmus humilis (Drury), coletados em Piracicaba, E. de S. Paulo. De cada indivíduo foi um testículo montado em orceina acética e o outro fixado em Allen-Bauer, incluido em parafina, cortado com 10-12 micra e os cortes coloridos com Hematoxilina de Heidenhain.

\section{LYBINDUS DICHROUS}

Espermatogônios - São providos de 13 cromossômios, ou seja, de um heterocromossômio e 6 pares de autossômios. (Fig. 1). O heterocromossômio não se deixa reconhecer nas placas metafásicas. Os autossômios constituem 5 pares de elementos curtos e aproximadamente do mesmo tamanho e um par de grandes elementos, alongados e grossos. Estes últimos exibem uma leve constrição mediana, que se pode observar no final da prófase. Essa constrição, ao contrário do que poderia parecer, não corresponde a um centrômero mediano, porquanto, do comêço ao fim da anáfase, as metades que resultam da divisão longitudinal daqueles autossômios, à semelhança do 
que se passa com as provenientes da divisão dos demais, movem-se, para os pólos, paralelamente ao equador da célula.

Espermatócitos primários - Além do heterocromossômio, exibem 5 tétrades curtas e pouco diferentes entre si e uma grande tétrade correspondente aos dois autossômios alongados assinalados nos espermatogônios. (Fig. 4).

Dadas as avantajadas dimensões da tétrade maior, pôde-se acompanhar a sua formação e estudar o seu comportamento. Verificou-se, então, que ela resulta de uma indubitável parassinapse dos dois grandes autossômios. Desde antes da diacinese pode-se observar que a tétrade maior, que geralmente se mostra com o formato de cruz, (Fig. 6), também se apresenta como um elemento alongado, fendido longitudinalmente e constituido por dois braços simétricos, em cuja zona de contato não chegam a se formar os braços transversais da cruz assinalada em outras células. (Fig. 5). A forma final com que essa tétrade se apresenta na metáfase, corresponde ao seu modo de formação: cruz de braços transversais mais alongados ou mais abreviados ou um espêsso corpo cilindróide constituido por dois braços separados por um espaço transversal, por onde os dois braços se podem dobrar em ângulo mais ou menos aberto. Ao orientar-se, a tétrade em questão se dispõe no equador da célula, de tal modo, que os braços transversais da cruz fiquem no plano equatorial e os braços longitudinais voltados para pólos opostos. (Fig. 18). Se ela não exibe a forma de cruz, orienta-se paralelamente ao eixo do fuso. (Fig. 17). Caso se ache dobrada pela região mediana, pode dispor-se no plano do equador, com os braços sobrepostos voltados para os pólos.

$\mathrm{Na}$ anáfase, qualquer que seja a posição da tétrade, ela se divide pela região mediana e os cromossômios se encaminham para os pólos com a forma típica de um espêsso $V$, resultante do afastamento das extremidades acêntricas. (Figs. 7 e 19).

O heterocromossômio se divide na primeira divisão, de sorte que os espermatócitos secundários são todos providos de 7 cromossômios $(6 \mathrm{~A}+\mathrm{X})$.

Espermatócitos secundários - Terminada a divisão do espermatócito primário, os cromossômios, dentro de algum tempo, se arranjam, de novo, em metáfase. (Figs. 8 e 9). Os autossômios se dispôem em círculo, ficando o heterocromossômio na parte central. $O$ autossômio maior se apresenta com a forma de $\mathrm{V}$ de ramos mais ou menos aproximados e com o vértice voltado para fora. O heterocromossômio, que de todos é o menor elemento, mostra certa afinidade para com as extremidades abertas daquele autossômio, ficando muito próximo, ou mesmo se pondo em contato com uma delas. As vistas laterais 
mostram que a forma de $\mathrm{V}$ não é a única sob a qual se apresenta o autossômio maior. (Fig. 10). Também pode mostrar-se como um X, de ramos opostos iguais ou desiguais. Na anáfase, se o cromossômio metafásico tinha a forma de V, êle simplesmente se divide, aumentando cada vez mais a abertura, até que os dois ramos se libertem. (Figs. 20, 21 e 22). Estes se encaminham, então, para os pólos, movendo-se paralelamente ao eixo do fuso. (Fig. 22). Se o cromossômio metafásico tinha a forma de $X$, êle primeiramente se converte em $\mathrm{V}$ e depois se comporta como precedentemente. (Figs. 10 e 11).

O cromossômio maior, como vimos, chega aos pólos, na primeira divisão, com a forma de V. Do final dessa divisão, até a metáfase da segunda, aquêle cromossômio se transforma em $\mathrm{X}$ e depois, novamente, em $\mathrm{V}$, podendo conservar a primeira daquelas formas até o início da anáfase secundária. Este procedimento, de extraordinária significação, será esclarecido ao discutirmos os fatos aqui tratados.

O heterocromossômio permanece no equador da célula por algum tempo, enquanto os autossômios se dirigem para os pólos. (Fig. 11). Passa, indiviso, para um dos espermatídios resultantes, de sorte que apenas a metade dos espermatozóides produzidos serão portadores dêsse cromossômio.

Células somáticas da fêmea - Nas metáfases das células das paredes dos ovários encontram-se 14 cromossômios, sendo que os dois grandes autossômios apresentam o mesmo aspecto com que os conhecemos nos espermatogônios. (Fig. 2).

Tipo sexual - Macho XO, fêmea XX.

Cromossômios supernumerários - Um macho foi encontrado, cujos espermatogônios eram providos de 15 cromossômios. (Fig. 3). Ao contrário do esperado, os espermatócitos primários exibiam, alem do heterocromossômio, mais 8 elementos, 2 dos quais eram monovalentes. (Fig. 12). Estes passavam ao acaso para um ou outro pólo, de sorte que espermatócitos secundários com $7(6 \mathrm{~A}+\mathrm{X})$, com $8(6 \mathrm{~A}+\mathrm{X}+\mathrm{S})$ e com 9 $(6 \mathrm{~A}+\mathrm{X}+\mathrm{S}+\mathrm{S})$, foram produzidos. (Figs. 13, 14 e 15).

\section{EURYOPHTHALMUS HUMILIS}

O único macho estudado desta espécie trouxe completa confirmação de tudo quanto antes escrevera e concluira relativamente a Euryophthalmus rufipennis Laporte (PIZA, 1946a).

Os espermatogônios são providos de 13 cromossômios alongados e grossos, pouco diferentes entre si. (Fig. 23). Apresentam-se direitos ou ligeiramente recurvados e se orientam com a maior dimensão no plano do equador. 
As metáfases primárias, em vista polar, exibem 6 tétrades aproximadamente do mesmo tamanho e um heterocromossômio um pouquinho menor, situado do lado de fora, a pequena distância. (Fig. 24). As vistas laterais mostram que as tétrades têm a forma típica de halteres e se orientam paralelamente ao eixo do fuso, de tal sorte, que a cintura mediana fique no plano do equador. (Fig. 25). O heterocromossômio mostra-se então bem mais curto que as tétrades autossomais, leva mais tempo para se orientar, mas acaba se arranjando no equador, de idêntica maneira. Nenhuma tétrade, nem na diacinese, nem mesmo antes, tem forma de cruz. $\mathrm{Na}$ diacinese e na metáfase as tétrades podem se mostrar direitas ou mais ou menos dobradas pela região mediana. No comêço da anáfase, porém, as tétrades dobradas se abrem, distendendo-se sôbre os meridianos do fuso. $O$ heterocromossômio divide-se e movimenta-se sincronicamente com os autossômios.

Dividido o espermatócito primário pela região equatorial. os cromossômios dos espermatócitos secundários se afastam, readquirem a forma de halteres e se arranjam em um plano, de jeito que o seu maior eixo fique nesse plano. (Fig. 28, Foto 1 ).

Com o restabelecimento da bipolaridade nos espermatóci-

tos secundários, os cromossômios se orientam, girando de 90 graus ou de quanto for necessário, para se colocarem com o seu maior eixo paralelamente ao eixo do fuso. $\mathrm{E}$ assim se instala a placa metafásica da segunda divisão. (Fig. 26, Foto 3). Aqui, o heterocromossômio, ao contrário do que se observa na metáfase primária, ocupa o centro do círculo formado pelos autossômios.

A anáfase secundária difere da primária apenas pelo comportamento do heterocromossômio. Este elemento, que passa sem se dividir para um dos pólos, permanece longo tempo no equador, enquanto os autossômios se vão dêle afastando. Porém, as suas extremidades acompanham as placas anafásicas, assumindo êle a forma de um fuso, que cada vez mais se alonga. (Fig. 27, Fotos 2 e 3). Acaba, finalmente, alcançando um dos pólos e se incorporando ao núcleo formado nesse pólo. (Foto 4).

Espermatídios com 6 cromossômios e com $7(6 \mathrm{~A}+\mathrm{X})$ podem ser com facilidade reconhecidos logo que se constitui a membrana nuclear.

\section{DISCUSSAO}

Numa série de publicações tenho procurado provar que os cromossômios dos Hemípteros são providos de um centrômero em cada extremidade. (PIZA 1945, 1946a, 1946b, 1946c). Ao es- 
tudar agora a meiose de duas espécies ainda não investigadas, Lybindus dichrous Stal (Coreidae) e Euryophthalmus humilis (Drury) (Pyrrhocoridae), encontro mais alguns fatos de grande valor demonstrativo, que passarei a discutir e esclarecer.

Lybindus possui um par de autossômios muito maiores que os demais, cujo comportamento, nas duas divisões meiósicas, prova a dicentricidade cromossômica, no sentido que lhe venho dando.

$\mathrm{Na}$ diacinese e nos estádios que de pouco a precedem, os autossômios em questão constituem uma tétrade, que tanto se pode apresentar em forma de cruz, (Fig. 6), como na de um corpo alongado, cilindróide, direito ou dobrado ao meio, sem nenhum vestígio dos braços laterais que por ventura tivessem existido (Fig. 5). Como em fases que se correspondem pelo aspecto das outras tétrades, a maior pode ou não se constituir em cruz, conclui-se que o aspecto alongado não resulta de uma cruz que se tenha distendido no sentido de desfazer os braços transversais e sim, que a formação dêsses braços é facultativa. Tais sejam as condições e os braços transversais da cruz se formam ou a tétrade permanece alongada.

Em alguns Hemípteros, todas as tétrades têm forma de cruz (Leptoglossus gonagra); em outros (Euryophthalmus ruripennis, E. humilis, Alydus calcaratus), nenhuma tétrade forma cruz; em outros, finalmente, algumas formam cruz e outras não (Diactor bilineatus). A formação de tétrades em cruz é, pois, um caráter específico.

Nas espécies em que apenas uma ou algumas tétrades formam cruz, poderemos considerar o fenômeno como sendo devido a particularidades individuais dos cromossômios. Neste último caso, a formação de cruz pode ser obrigatória ou facultativa como em Lybindus. Em geral se verifica que são os autossômios maiores que produzem as tétrades em cruz.

A gênese das configurações em cruz pode ser facilmente compreendida, assumindo-se, como tem sido feito pelo presente autor, que os cromossômios dos Hemípteros são iso-cromossômios. (PIZA 1946a). Nessas condições, o pareamento tanto se pode dar ao longo do corpo dos parceiros associados, como entre segmentos do mesmo cromossômio, o que depende da espécie, ou, dentro da espécie, da individualidade dos cromossômios. (Fig. 29).

A tétrade grande de Lybindus ao dividir-se, origina dois cromossômios anafásicos com a forma típica de $\mathrm{V}$ dos cromossômios ortodoxos providos de centrômero mediano. (Figs. 7 e 19). Esse fato, inteiramente contrário à idéia de inserção difu- 
sa, sugere a possibilidade da existência de um centrômero único, localizado no meio dos cromossômios. A isso, porém, se opōe o fato dêsses cromossômios, que nos espermatogônios se apresentam como longos bastonetes, moverem-se para os pólns, paralelamente ao plano equatorial, sem jamais se dobrarem em forma de $V$.

Os Vs da anáfase primária ganham os pólos nessa forma. Não seria de admirar, por conseguinte, que êles a conservassem e com ela aparecessem nas metáfases secundárias, como aliás acontece. (Figs. 8, 9, 10, 20 e 21). Porém, verifica-se, pelas vistas polares, que o vértice do $V$ se acha voltado para o lado de fora, ao contrário do que seria de esperar, se os centrômeros se encontrassem nêle localizados, tal como se constatou nas anáfases primárias. (Figs. 8 e 9). As vistas laterais mostram claramente que o cromossômio em $V$ não possui centrômeros no vértice e sim um centrômero na extremidade de cada ramo: (Figs. 20 e 21), justificando plenamente a posição por êle tomada na metáfase secundária, com a abertura dirigida para a parte central da placa. Aumentando cada vez mais a abertura, o V acaba se dividindo e os seus ramos se encaminham para pólos opostos, movendo-se paralelamente ao eixo do fuso. (Figs. 11 e 22).

O comportamento contraditório do V na primeira e na segunda divisão, revelando numa a presença de centrômeros nn vértice e noutra, de um centrômero na extremidade de cada ramo, seria assás embaraçoso, caso tôdas as etapas que conduzem de uma situação a outra, não tivessem sido encontradas. De fato, do final da primeira divisão até a metáfase da segunda, o cromossômio em V começa a se abrir pelo vértice, em virtude da repulsão dos centrômeros aí localizados. (Cf. PIZA 1946c). (Fig. 30). Quanto mais se afastam os centrômeros, mais diminui, do lado oposto, a primitiva abertura. O cromossômio vai então adquirindo a forma de um $\mathrm{X}$ de ramos opostos desiguais, que depois se igualam, para mais tarde novamente se desigualarem e assim, progressivamente, até se converter outra vez em um V, que será invertido. (Fig. 30).

$\mathrm{Se}$ a inversão completa do $\mathrm{V}$ não se realizar antes da metáfase, o cromossômio se orientará, no equador da célula, com a forma de um $X$ de ramos iguais ou desiguais. (Fig. 10). Ao iniciar-se a anáfase, $0 \mathrm{X}$ se transforma em $\mathrm{V}$ e depois os dois ramos do $V$ se separam e se dirigem para os pólos correspondentes, movendo-se paralelamente ao eixo do fuso. (Figs. 10, $11,20,21$ e 22).

$O$ fato dos cromossômios que constituem os ramos do V não se libertarem em consequência da repulsão dos centrôme- 
ros localizados no vértice, prova a existência de quiasmas, que se deslocando da extremidade cêntrica para a extremidade acêntrica, dão origem às figuras em forma de $X$. (Fig. 30).

Essa complicada manobra que acabámos de discutir, oferece-nos a prova crucial de que os cromossômios dos Hemípteros são providos de um centrômero em cada extremidade.

Outra prova de dicentricidade encontramos na conduta do heterocromossômio de Euryophthalmus humilis. A semelhança do que já foi constatado e descrito em Euryophthalmus rufipennis (PIZA 1946a) e em outras espécies (PIZA 1.c), o heterocromossômio, que na primeira divisão se orienta e se comporta como uma tétrade autossomal, na segunda passa indiviso para um dos espermatídios. Na metáfase secundária aquêle cromossômio se coloca no centro do círculo - formado pelos autossômios, estabelecendo conexão fibrilar com ambos os pólos. Permanece no equador da célula por um certo tempo, enquanto os autossômios se vão afastando. As suas extremidades, porém, acompanham as placas que se distanciam, de sorte que êle se var convertendo num fuso cada vez mais alongado. (Fig. 27, e Fotos 2 e 3).

Ora, quer-me parecer, que um tal comportamento, de um cromossômio que não se vai dividir, já discutido em outras espécies, não pode ser compreendido, a não ser que se admita a presença de um centrômero ativo em cada uma das extremidades que se deslocam com os autossômios para os pólos.

Um outro ponto que merece a melhor das atençōes referese ao comportamento dos cromossômios no periodo que vai do final da primeira divisão à metáfase da segunda. E' nesse período que os cromossômios se reorganizam e se orientam para a segunda divisão. Havendo chegado aos pólos com a forma de meios-halteres, novamente adquirem a forma de halteres completos das tétrades típicas. Isso se verifica em virtude da repulsão dos centrômeros.

Concluida a primeira divisão, com ela se termina a bipolaridade orientadora dos movimentos dos cromossômios. Antes que os espermatócitos secundários se tornem, por seu turno, bipolares, os cromossômios se libertam, por algum tempo, das influências cinéticas a que estiveram antes sujeitos. Nessa nova situação, readquirindo a forma de halteres, encontram a sua posição de equilíbrio, deitando-se num plano que teria por centro a projeção do pólo que os orientara. Enquanto os dois centrossômios dos espermatócitos secundários se encontrarem muito próximos entre si, é bastante provável, caso êles exerçam alguma influência sôbre os centrômeros, que auxiliem os cromossômios que ainda não o fizeram, a se distender horizontal- 
Provas cruciais da dicentricidade dos cromossômios dos Hemípteros 163

mente sôbre aquêle plano. Entretanto, não é obrigatório que todos os cromossômios se distendam ao mesmo tempo. Enquanto uns já alcançaram a sua posição de equilíbrio, outros podem se encontrar ainda numa posição perpendicular ou oblíqua com relação ao plano em que os primeiros se encontram. Tal seja a rapidez com que os centrossômios se afastem para restabelecer a bipolaridade, e os cromossômios que ainda se não deitaram, nem cheguem a fazê-lo, passando diretamente da posição em que se achavam, para a posição orientada da metáfase. Em Euryophthalmus humilis, a bipolaridade leva um certo tempo para se restabelecer, razão pela qual todos os cromossômios se deitam. (Fig. 28 e Foto 1). Além disso, o fato de alguns cromossômios se apresentarem em posição perpendicular ou oblíqua, pode apenas significar, que no momento em que a célula foi fixada êles não tinham ainda tomado a posição distendida dos demais. O certo é, que os aspectos que antecedem à bipolaridade e por conseguinte à orientação, simulam uma metáfase em que alguns cromossômios se apresentam deitados ou de pé, com relação ao plano que ocupam.

$\mathrm{Na}$ espermatogênese dos Hemípteros estudados pelo autor, encontram-se inúmeras metáfases primárias em que um ou mais cromossômios se acham deitados no plano equatorial, enquanto os outros se apresentam dispostos paralelamente ao eixo do fuso, ou seja, com as extremidades voltadas para os pólos. Isso, ao contrário do que pensa HELENIUS (1952), não significa dois modos distintos de orientação: auto-orientação e co-orientação, respectivamente. A grande duração da metáfase primária está em. relação com a dẹmora que levam os cromossômios para se orientar. Os que se encontram deitados são exatamente aquêles que ainda se não orientaram. E' possível que os cromossômios mais rebeldes só se orientem quando as fôrças que atuam na anáfase iniciem a sua atividade. Nessa ocasião, pode-se com facilidade constatar que tôdas as tétrades se acham co-orientadas. A auto-orientação nas metáfases primárias, pois, apenas significa que as tétrades ainda não experimentaram a influência orientadora dos pólos.

NORONHA-WAGNER e DUARTE DE CASTRO (1952b), estudando a meiose de Luzula nemorosa, constataram que o comportamento dos cromossômios daquela planta, em ambas as divisões, é exatamente aquêle descrito por PIZA para os Hemípteros. Fugindo, porém, à evidência dos fatos, aquêles autores imaginaram, para explicar o fenômeno, um esquema baseado na suposição de que os cromossômios fôssem providos de cen- 
trômero difuso, que reputo inteiramente inadequado. De fato, se tudo indica, como reconhecem os autores, que os cromossômios se orientam e se dividem como se as açōes polares se exercessem apenas nas extremidades, para que apelar para assunções destituidas de suporte objetivo ? Realmente, se a única indicação que pode levar à idéia de inserção difusa é a maneira dos cromossômios se orientarem e se moverem paralelamente ao equador do fuso, não se deve pensar em tal, uma vez que os cromossômios se orientam e se movem paralelamente ao eixo do fuso. Além disso, solicitados para os pólos por fôrças que atuam ao longo de todo o corpo, cromossômio algum seria mecanicamente capaz de assumir uma posição perpendicular ao plano do equador.

Nem o esquema, nem as fotografias dos autores sugerem que os cromossômios em metáfase ou anáfase tenham resultado de um pareamento primário dos próprios braços, seguido de um pareamento secundário dos dois membros de cada par. Tudo indica, pelo contrário, que os homólogos se parearam, como nos Hemípteros, ao longo do seu comprimento. O pareamento dos braços dos iso-cromossômios satisfaria às fôrças de perea. mento e näo mais haveria fôrças a que apelar para a realização de novo pareamento, ainda mais que os cromossômios homólogos poderiam se encontrar muito afastados um do outro. A idéia de um pareamento de braços seguido de um pareamento de cromossômios levou os autores a complicar os acontecimentos, admitindo pareamento também na segunda divisão.

A evidente dicentricidade dos cromossômios da Luzula nemorosa permite simplificar bastante o esquema dos autores, aceitando, como se dá com os Hemípteros, que, concluida a primeira divisão, há uma soldadura pelas extremidades recémfraturadas, de hemi-cromossômios e não de hemi-cromatídios independentemente. $\mathbf{E}$ assim os cromossômios que reaparecem na metáfase II exibem os seus dois cromatídios, não sendo necessário apelar para um segundo pareamento de cromatídios que se haviam separado antes. Concluida a segunda divisão, os hemi-cromatídios que se dirigem para os pólos de novo se unem pela extremidade acêntrica, regenerando os iso-cromossômios.

Os cromossômios dicêntricos orientam-se e movimentamse de duas maneiras na meiose : se os dois membros de cada bivalente não se acham presos um ao outro por quiasmas e os centrômeros se repelem desde muito cedo, êles se orientam paralelamente ao equador e se movem com essa orientação ( $\mathrm{Ti}$ tyus) (Fig. 31); se, pelo contrário, os centrômeros năo se repelem, quer porque a fôrça de pareamento não se tenha ainda 
relaxado, (Fig. 32), ou porque os dois parceiros se encontram amarrados por quiásmas, (Fig. 33), os bivalentes se orientam e os cromossômios se movem paralelamente ao eixo do fuso ( $\mathrm{He}-$ mipteros). E isso, porque? Simplesmente porque não havendo repulsão das extremidades correspondentes, elas se comportam como se apenas um centrômero se achasse nelas presente. Nessas condições, a atuação dos pólos criaria um binário que mais cedo ou mais tarde obrigaria o conjunto a girar até alcançar a sua posição de equilíbrio, o que se daria quando o seu eixo se dispuzesse paralelamente ao eixo do fuso. (Figs. 34 e 35 ).

Os univalentes dicêntricos, se já tiverem os centrômeros divididos por ocasião da metáfase, orientam-se e movem-se paralelamente ao equador. (Espermatogônios de Tityus e de Hemípteros). Se os centrômeros se mantiverem indivisos, porém ativos, o cromossômio se orienta paralelamente ao eixo do fuso. (Figs. 34 e 35). (Heterocromossômio de alguns Hemípteros na divisão heterocinética); quando inativos, o cromossômio não se orienta, permanecendo em qualquer ponto da célula. (Heterocromossômio de certos Hemípteros na divisão heterocinética).

O univalente de Luzula campestris (NORONHA-WAGNER e DUARTE DE CASTRO, 1952a), possuindo cinetocores indivisos, porém ativos, orienta-se, conforme o esperado, paralelamente ao eixo do fuso.

\section{RESUMO}

Estudando a meiose de dois Hemípteros, Lybindus dichrous (Coreidae) e Euryophthalmus humilis (Pyrrhocoridae), encontra o A. novas provas a favor da existência de um centrômero em cada extremidade dos cromossômios dos Insetos daquela Ordem.

Seguindo o comportamento de um par de grandes autossômios de Lybindus, pôde constatar que na primeira divisão dos espermatócitos, a tétrade por êles formada divide-se transversalmente ao meio, dando dois cromossômios anafásicos em forma de $V$, que se dirigem para os pólos com o vértice para a frente. Do final da primeira divisão até a metáfase da segun$\mathrm{da}$, os centrômeros localizados no vértice do $\mathrm{V}$ se afastam, fazendo os quiasmas ali existentes correr para as extremidades opostas, o que transforma o $\mathrm{V}$ em X. Ao atingirem os quiasmas as extremidades acêntricas, o $\mathrm{X}$ de novo se converte em $\mathrm{V}$. O V 
da metáfase secundária, pois, difere do $\mathrm{V}$ da anáfase primária, por ser invertido, isto é, por apresentar os centrômeros na extremidade dos braços e não mais no vértice, como naquela. Fica assim demonstrada a abertura dos cromossômios a partir das extremidades cêntricas, para regenerar a forma de halteres com que se apresentam na metáfase secundária, da maneira postulada por PIZA.

Em Euryophthalmus humilis, verificou-se, mais uma vez, que o heterocromossômio, nos espermatócitos secundários, orienta-se paralelamente ao eixo do fuso, acompanhando, com as extremidades, as placas anafásicas que se dirigem para os pólos.

O autor discorda da interpretação de NORONHA-WAGNER e DUARTE DE CASTRO, relativamente ao comportamento dos cromossômios na meiose de Luzula nemorosa.

\section{LITERATURA}

Encontra-se no final do texto inglês.

\section{EXPLICAÇAO DAS FIGURAS}

\section{Lybindus dichrous}

Fig. 1 - Espermatogônio com 13 cromossômios.

Fig. 2-Célula somática da fêmea com 14 cromossômios.

Fig. 3 - Espermatogônio com 15 cromossômios, 2 dos quais são supernumerários.

Fig. 4-Vista polar da metáfase primária, vendo-se ao alto a tétrade maior dobrada em espêsso $\mathrm{V}$.

Fig. 5-Um dos aspectos da tétrade maior algum tempo antes da metáfase primária.

Fig. 6-Outro aspecto da mesma tètrade em fase correspondente à da Fig. 5 .

Fig. 7 - Os componentes da tétrade maior numa vista lateral da anáfase primária.

Figs. 8 e $9-$ Duas vistas polares da metáfase secundária, vendo-se o cromossômio maior em forma de $\mathrm{V}$ com a abertura voltada para dentro.

Fig. 10 - Dois aspectos do cromossômio maior em vista la teral da metáfase secundária. 
Fig. 11 - Vista lateral da anáfase secundária, vendo-se, à esquerda, os cromossômios maiores movendo-se paralelamentes ao eixo do fuso.

Fig. 12 - Vista polar da metáfase primária com 2 cromossômios super-numerários.

Figs. 13, 14 e 15-Vista polar de metáfases secundárias, respectivamente com 7,8 e 9 cromossômios.

Figs. 16-19, Interpretação da estrutura e do comportamento dos cromossômios maiores na primeira divisão: 16, univalente com um centrômero em cada extremidade; 17 e 18, dois aspectos assumidos pela tétrade maior numa vista lateral da metáfase primária; 19 , vista lateral dos componentes da tétrade maior na anáfase primária.

Figs. 20-22, Interpretação do comportamento dos cromossômios maiores na segunda divisão : 20 e 21, dois aspectos da metáfase em vista lateral; 22, vista lateral da anáfase.

\section{Euryophthalmus humilis}

Fig. 23 - Espermatogônio com 13 cromossômios.

Fig. 24 - Vista polar da metáfase primária, vendo-se, ao alto, o heterocromossômio.

Fig. 25 - Vișta lateral da metáfase primária, vendo-se, à direita, o heterocromossômio.

Fig. 26 - Vista polar da metáfase secundária, vendo-se an centro, o heterocromossômio.

Fig. 27 - Vista lateral da anáfase secundária, vendo-se o heterocromossômio distendido entre as duas placas.

Fig. 28 - Os cromossômios do espermatócito secundário, antes da orientação, deitados em um plano.

Fig. 29 - Esquema mostrando como podem parear-se os isocromossômios para originar tétrades em forma de hạlteres ou de cruz.

Fig. 30-Aspectos assumidos pelo cromossômio maior de Lybindus, do final da primeira divisão até a metáfase da segunda (em cima) e sua interpretação (em baixo).

Figs. 31-35, Explicação da maneira pela qual se orientam as tétrades formadas por cromossômios dicêntricos. (Veja o texto). 


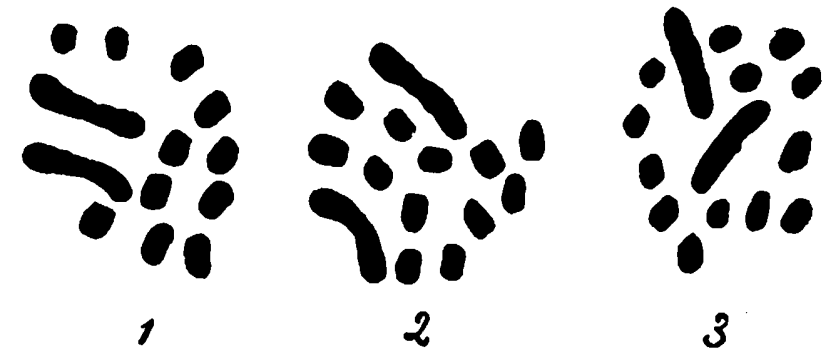

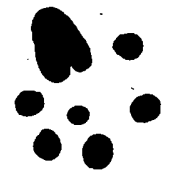

4

$0_{0}^{\circ}$

8

$\int_{0}^{\circ}$

12

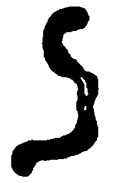

5

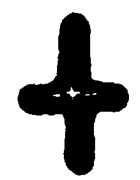

61
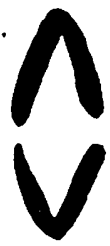

7

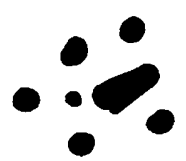

9
13

10 ins

hos

11

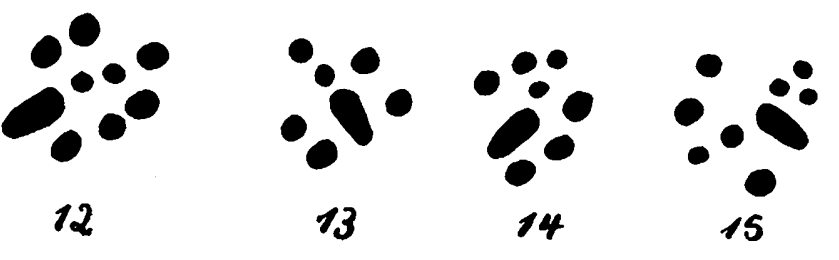


Provas cruciais da dicentricidade dos cromossômios dos Hemípteros 169
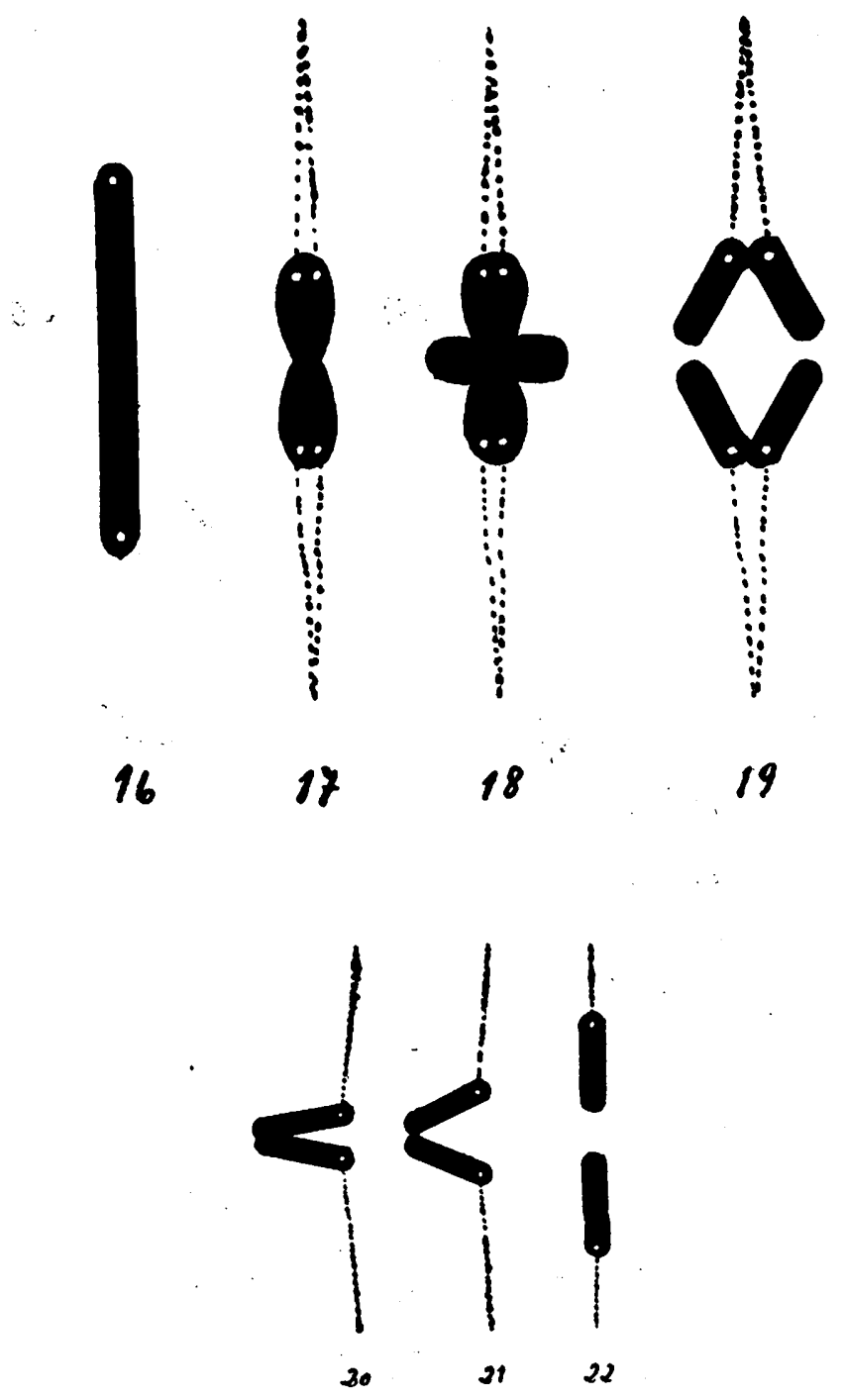


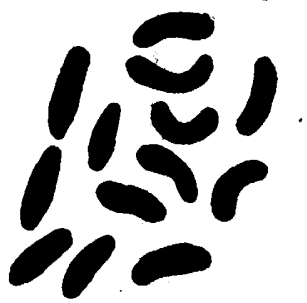

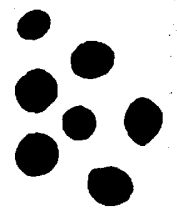

24

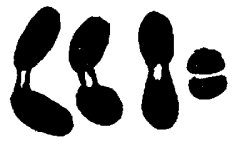

25

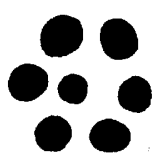

26

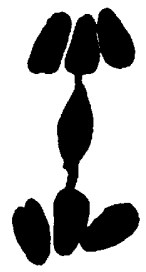

27

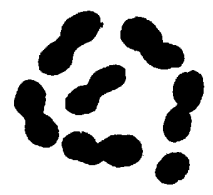

28

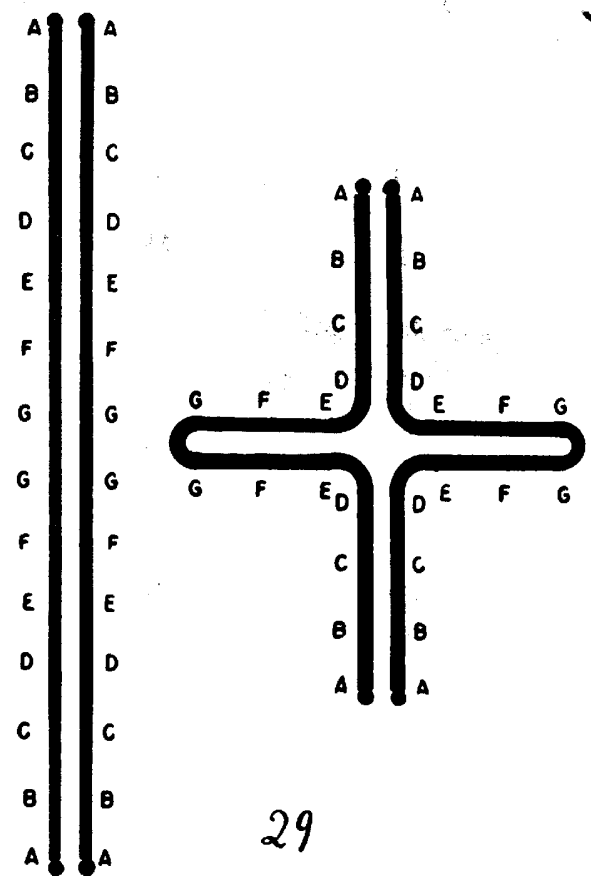



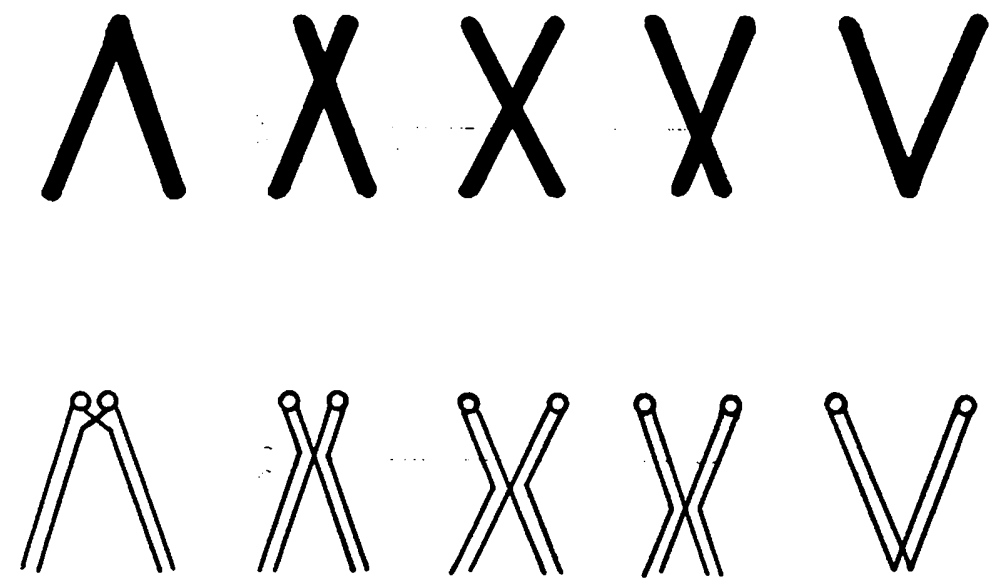

30 
m
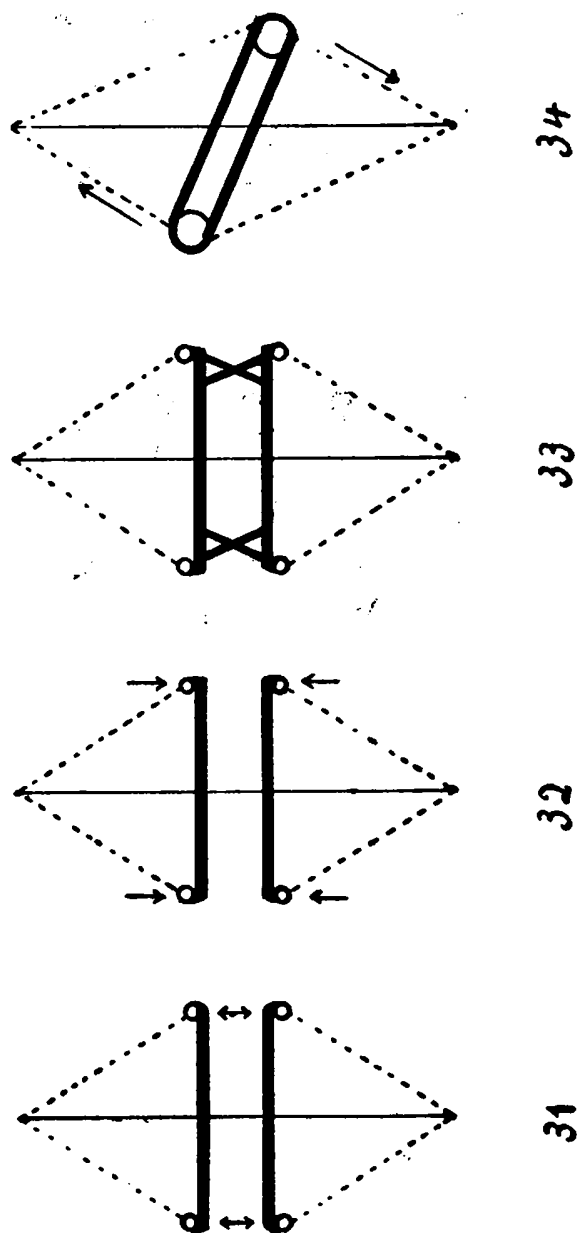


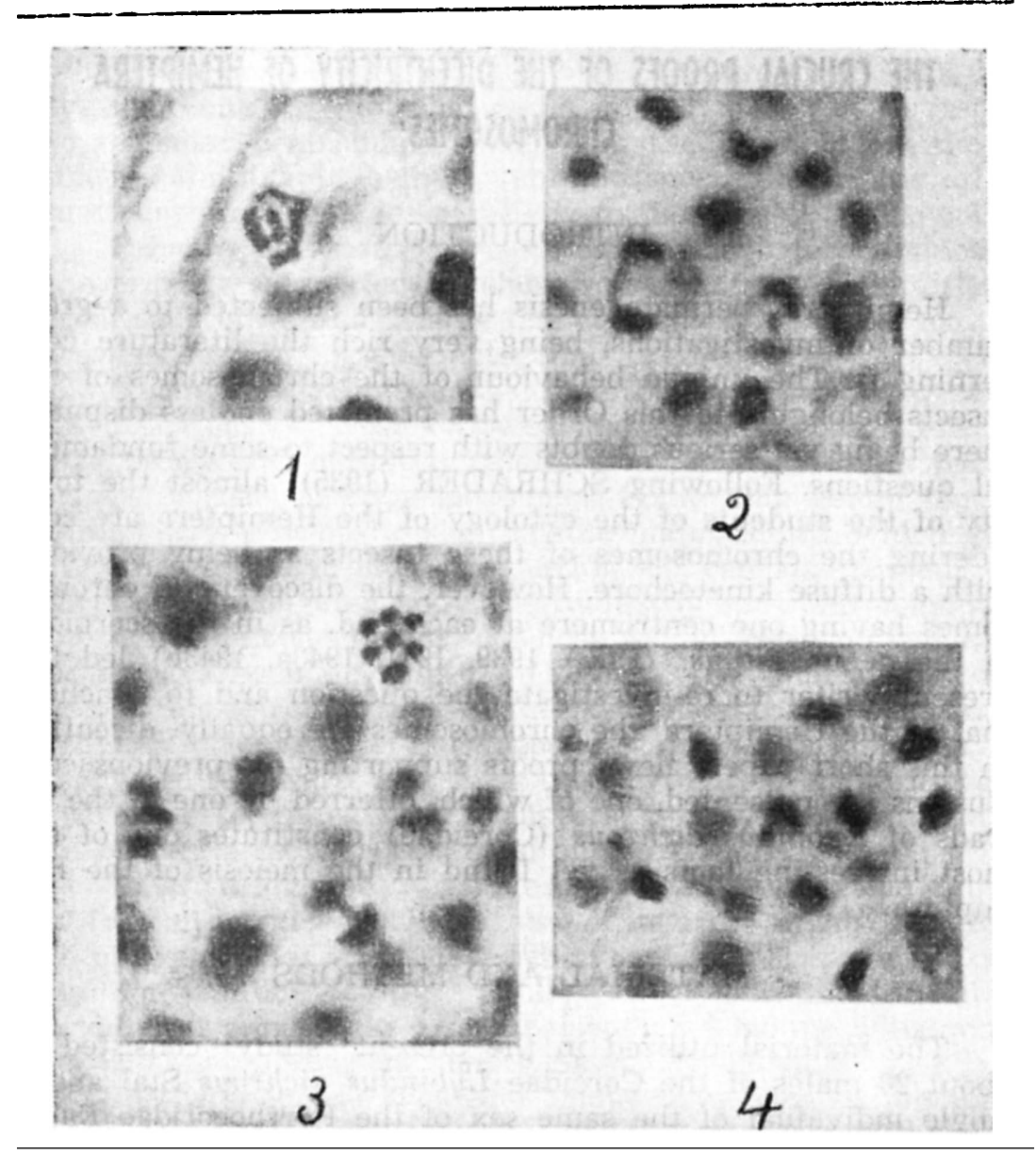

\section{EXPLICAÇAO DAS FOTOGRAFIAS}

\section{Euryophthalmus humilis}

1 - Posição assumida pelos cromossômios antes da metáfase secundária.

$2-$ Duas vistas laterais da anáfase secundária; vendo-se o heterocromossômio distendido entre as duas placas.

3-Metáfase secundária com o heterocromossômio no mein (em cima) e anáfase secundária com o heterocromossômio distendido entre as duas placas (em baixo).

4-Anáfase secundária com o heterocromossômio nas proximidades de um dos pólos (no centro).

(Aumento : cêrca de $\mathrm{x}$ 1000). 


\section{THE CRUCIAL PROOFS OF THE DICENTRICTYY OF HEMIPTERA CHROMOSOMES}

\section{INTRODUCTION}

Hemiptera spermatogenesis has been subjected to a great number of investigations, being very rich the literature concerning it. The unique behaviour of the chromosomes of the Insects belonging to this Order has promoted endless disputes, there being yet serious doubts with respect to some fundamental questions. Following SCHRADER (1935), almost the totality of the students of the cytology of the Hemiptera are considering the chromosomes of these Insects as being provider with a diffuse kinetochore. However, the discovery of chromosomes having one centromere at each end, as in the scorpions of the genus Tityus, (PIZA 1939, 1941, 1943a, 1943b) led the present writer to re-investigate the question and to conclude that in the Hemiptera the chromosomes are equally dicentric. In this short paper new proofs supporting his previous conclusions are presented, one of which, offerred by one of the tetrads of Lybindus dichrous (Coreidae) constitutes one of the most interesting facts as yet found in the meiosis of the Hemiptera.

\section{MATERIAL AND METHODS}

The material utilized in the present study consisted of about 20 males of the Coreidae Lybindus dichrous Stal and a single individual of the same sex of the Pyrrhocoridae Euryophthalmus humilis (Drury), collected in Piracicaba, S. Paulo. One of the testes from each individual was mounted in acetic orsein and the other fixed in Allen-Bauer, embeded in paraffim, cut at 10-12 micra and the sections stained with Heidenhain's haematoxylin.

\section{LYBINDUS DICHROUS}

Spermatogonia - The spermatogonia are provided with 13 chromosomes, that is, with one heterochromosome and 6 pairs of autosomes. (Fig. 1). The former cannot be recognized in the metaphase plates. The latter constitute 5 pairs of short elements approximately the same size, and one pair of long and thick elements. These show an indistinct median constriction 
which can be observed at the end of the prophase. This constriction, contrarily to what could appear, does not correspond to a median centromere, since, from the beginning to the ending of the anaphase these chromosomes, like all the others, move towards the poles parallelly to the equator of the cell.

Primary spermatocytes - Besides the heterochromosome the primary spermatocytes show $\mathbf{5}$ short tetrads little different from each other and a large one corresponding to the long au. tosomes seen in the spermatogonia. (Fig. 4).

Given the advantageous dimensions of the major tetrad its formation could be followed and its behaviour studied. It was then verified that it results from an indubitable parasynapsis of the two large autosomes. Some time before the diakinesis it is possible to see that the major tetrad, which generally has the form of a cross, (Fig. 6), can also present the form of an elongated body longitudinally split and constituted by two symmetrical arms in whose joining point no transverse arms have been developed. (Fig. 5). The final shape with which this tetrad is seen in the metaphase corresponds to the manner in which it is developed : a cross with more or less elongated transverse arms or a thick cylindroid body formed by two arms separated by a transverse space whereby they can be bent in a more or less open angle.

In orienting, the tetrad in issue puts itself in the equator of the cell in such a way that two of its arms are always turned to opposite poles. (Fig. 18). When not cross-shaped it takes a position parallelly to the spindle axis. (Fig. 17). If bent at the middle, its superposed arms are separated by the equator plane passing through the joning point.

In the anaphase, whatever might have been its metaphase shape, the tetrad divides by the median region and the resulting parts move towards the poles in the form of a bulky $\mathrm{V}$ originated from the opening out of the acentric extremities. (Figs. 7 and 19).

The heterochromosome divides at the first division so that all the secondary spermatocytes are provided with 7 chromosomes $(6 \mathrm{~A}+\mathrm{X})$.

Secondary spermatocytes - The division of the primary spermatocytes once finished, the chromosomes, in a short time, form again a metaphase plate. (Figs. 8 and 9). The autosomes form a circle around the heterochromosome. The large one has the shape of a less open $\mathrm{V}$ having the vertex turned outwardly. The heterochromosome which is smaller than the autosomes shows some affinity to the open ends of the V-shaped autosome, 
appearing in their proximity or even in contact with one of them The side views show that the $V$ does not be the unique shape assumed by the major autosome. (Fig. 10). It can also appear with the form of an $\mathrm{X}$ of equal or unequal opposed arms. When V-shaped, it simply increases the opening till the complete separation of its arms, which go to the poles parallelly to the axis of the spindle, as can be seen in the anaphase. (Figs. 20, 21 and 22). When $X$-shaped, it is at first converted into $a V$ and then behaves as precedently. (Figs. 10 and 11).

As we have seen, the large autosome reaches the pole with the form of a V in the first division. From the end of the first division till the metaphase of the second one it is transformed into an $\mathrm{X}$, and again into a V, the former shape being sometimes kept till the anaphase of the second division. This highly significative behaviour will be explained in discussing the facts delt with in this paper.

The sex chromosome rests for some time in the equator of the cell while the autosomes are going to the poles. (Fig. 11). It passes undivided to one of the resulting spermatids, so that only the half of the spermatozoa produced are in possession of this chromosome.

Somatic cells of the female - In the metaphase of the cells in the walls of the ovaries 14 chromosomes have been found, the two large autosomes showing the same aspect seen in the spermatogonia. (Fig. 2).

Sexual type - The male is XO and the female XX.

Supernumerary chrmosomes - A male was found whose spermatogonia were provided with 15 chromosomes. (Fig 3). Contrarily to what was expected, the primary spermatocytes exhibited in addition to the heterochromosome, 8 elements, 2 of which showed to be univelents. (Fig. 12). The latter passed accidentally to the one or the other pole, so that secondary spermatocytes with $7(6 \mathrm{~A}+\mathrm{X})$, with $8(6 \mathrm{~A}+\mathrm{X}+\mathrm{S})$ and with $9(6 A+X+S+S)$ chromosomes have been produced (Figs. 13, 14 and 15).

\section{EURYOPHTHALMUS HUMILIS}

The single male studied of this species brought a full confirmation to all that has been reported and concluded relatively to Euryophthalmus rufipennis Laport. (PIZA 1946a).

The spermatogonia are provided with 13 elongated and thick chromosomes little different from each other. (Fig. 23). They are stretched or slightly curved and orient with the majo: axis in the plane of the equator. 
In polar view of the metaphase of the primary spermatocytes we see 6 tetrads approximately the same size and a sex chromosome a little smaller placed outsidely at a short distance. (Fig. 24). The side views show that the tetrads are typically dumbbell-shaped and orient parallelly to the spindle axis, in such a way that the median constriction is sectioned by the equatorial plane. (Fig. 25). The heterochromosome then shows to be much shorter than the tetrads, orients more slowly, but finishes by arranging itself in a like manner in the equator. None of the tetrads, in the diakinesis or even before, is crossshaped. In the diakinesis and metaphase the tetrads may be stretched or bent by the median region. In the beginning anaphase, however, the bent tetrads open themselves out and distend themselves on the meridians of the spindle. The heterochromosome divides and moves synchronously with the autosomes.

As soon as the primary spermatocyte is divided by the equatorial region, the chromosomes separate from each other, acquire again a dumbbell shape and arrange themselves in $a$ plane, in such a way that their major axis lies in this plane. (Fig. 28 and Photo 1).

With the reestablishment of the bipolarity in the secondary spermatocyte the chromosomes orient in that plane undergoing a rotation of 90 degrees or as many degrees as necessary in order to put their major axis parallelly to the spindle axis. In this manner the metaphase plate of the second division is established. (Fig. 26 and Photo 3). Now, the heterochromosome, contrarily to what happens in the primary metaphase, occuppies the center of the circle formed by the autcsomes. The secondary anaphase differs from the primary one only by the behaviour of the sex chromosome. This element, which passes undivided to one pole, stays for a time in the equator, while the autosomes are moving towards the poles. But its ends follow the anaphase plates, giving him in this way the shape of a more and more elongating spindle. (Fig. 27 and Photos 2 and 3). It finishes by reaching one of the poles, entering into the nucleus formend in this pole (Photo 4),

Spermatids with 6 and $7(6 \mathrm{~A}+\mathrm{X})$ chromosomes can be easily recognized as soon as the nuclear membrane is formed

\section{DISCUSSION}

In a series of papers I have tried to prove that Hemiptera chromosomes are provided with one centromere at each end. (PIZA 1945, 1946a, 1946b, 1946c). Studying now the meiosis of 
two species not yet investigated, namely, Lybindus dichrous (Coreidae), and Euryophthalmus humilis (Pyrrhocorridae), I have found some additional data of great demonstrative value, which I pass to discuss and explain.

Lybindus has one pair of autosomes much larger than the rest, whose behaviour in both meiotic divisions proves the dicentricity of chromosomes in the sense I am giving it.

In diakinesis and the little preceding stages the autosomes in question form a tetrad which may appear in the shape of a cross (Fig. 6), or of a cylindroid body stretched out or bent at the middle, without any vestige of lateral branches which could by chance have been previously formed. (Fig. 5). As in stages corresponding to each other by the aspect of the other tetrads, the larger one may or may not arrange itself into a cross, we conclude that the elongated shape it sometimes assumes does not result from a distended cross, the formation of transverse branches being a facultative affair. Under such or surch conditions the transverse arms are developed or the tetrad remains as an elongated body.

In some Hemiptera all the tetrads are cross-shaped (Leptoglossus ganagra); in others (Euryophthalmus rufipennis, $E$. humilis, Alydius calcaratus) none of them forms cross; and, in others, finally, some form cross and some do not. (Diactor bilineatus). The formation of cross-shaped tetrads is therefore a specific attribute of the chromosomes. In the species in which only one or some tetrads form cross we can consider such a phenomenon as being due to individual peculiarities of the chromosomes. In these cases the cross formation may be obligatory, or facultative as in Lybindus. Ordinarily are the longest autosomes that give rise to cross-shaped tetrads.

The genesis of cross configurations may be easily undertood if we assume, as the present writer has done, that Hemiptera chromosomes are iso-chromosomes. (PIZA 1946a). Under this assumption pairing may be alongst the entire body of the associated mates as well as between corresponding segments of the same chromosome, what depends upon the species or, within the species, upon the individuality of the chrromosomes. (Fig. 29).

The large tetrad of Lybindus gives rise to two anaphase chromossomes with the typical V-shape of the orthodox mediocentric chromosomes. Such a fact, in full contradiction to the idea of a diffuse insertion, fairly suggests the possibility of the existence of a single centromere localized at the middle of the chromosomes. To this, however, opposes the fact that these 
chromosomes, which in the spermatogonia, have the form of long rods, move towards the poles parallelly to the plane of the equator, never assuming the shape of a V.

The V-shaped chromosome of the primary anaphase reaches the corresponding pole just in this form. It would, therefore, not wonder, if it keeps this form, appearing with it in the secondary metaphase, as it really happens. (Figs. 8, 9, 10, 20 and 21). Polar views, however, show that the V-shaped chromosome always appears with the vertex turned outwardly, contrarily to what should be expected if the centromeres were placed there as in the primary anaphase. (Figs. 8 and 9). The side views clearly show that the chromosome does not have centromeres at the vertex, but one centromere at the end of each branch, (Figs. 20 and 21 ), justifying in this manner the position it takes in the metaphase, with the opening turned towards the center of the plate. Increasing more and more its opening the $\mathrm{V}$ finishes by dividing itself and its free arms go to opposite poles moving parallelly to the spindle axis. (Figs. 11 and 22).

The contradictory behaviour of the $\mathrm{V}$ in the first and the second division, showing in the one centromeres localized at the vertex, and in the other a single centromere at the extremity of each branch, should be puzzling enough if we had not found all the intermediate stages going from one situation to the other. In fact, from the end of the first division till the metaphase of the second one, the V-shaped chromosome begins to open itself out by the apex, due to the repulsion of the centromeres localized there. (Cf. PIZA 1946c). (Fig. 30). The more the centromeres go away from one another, the more the opening at the opposite side is reduced. The chromosome, then, finishes by acquiring the form of an inverted V. (Fig. 30). If the process of inversion of the $\mathrm{V}$ does not end before the establishment of the secondary metaphase, then the chromosome orients itself in the equator of the spindle still in the form of an $X$ of equal or unequal arms. (Fig. 10). But, as soon as the anaphase movement starts, the $X$ is transformed into a $V$ whose branches separate afterwards and move to opposite poles parallelly to the axis of the spindle. (Figs. 10, 11, 20, 21 and 22).

The fact that the chromosomes forming the $\mathrm{V}$ do not freed from one another as the centromeres placed side by side in the vartex separate, but remain united at a moving point, proves the existence of chiasmata, that running from the centric. to the acentric extremity, give rise to the $\mathrm{X}$-shaped configura. tions. (Fig. 30). 
This complicated manoeuvre we have discussed and explained affords the crucial proof that the Hemiptera chromosomes are provided with one centromere at each end.

Another proof of dicentricity is found in the behaviour of the heterochromosome of Euryophthalmus humilis. Similarly to what was already reported with regard to Euryophthalmus rufipennis (PIZA 1946a) and to other species, (PIZA l.c.), the heterochromosome, which in the primary division behaves like an autosomal tetrad, passes undivided to one of the spermatids. In the secondary metaphase that chromosome is seen in the center of the circle formed by the autosomes, wherefrom it establishes fibrilar connexions with both poles. While the anaphase plates are moving towards the poles it remains for some time motionless in the equator of the cell. Its extremities alone accompany the autosomes, so that, in short, it acquires the shape of a more and more alongated spindle. (Fig. 27 and Photos 2 and 3). It seems to me that such a behaviour of a chromosome which does not divide, already discussed helsewhere, cannot be understood without admitting the presence of an active centromere at each moving end.

A question which deserves the best of our attention refers to the behaviour of the chromosomes in the period extending from the end of the primary division of the spermatocytes to the secondary metaphase. It is in this period that the chromosomes reorganize and orient themselves for the second division. Reaching the poles with the form of half-tetrads, they acquire again the dumbbell-shape of the typical tetrads. This is due to the repulsion of the centromeres. With the terminus of the first division disappears the bipolarity of the cell responsible for the oriented movement of the chromosomes. Before the secondary spermatocytes become by their turn bipolarized, the chromosomes are for a while freed from the kenetic influence they were subjected to. Reassuming their dumbbell-shape in this new situation, they find their equilibrium in the cellular medium by lying flattly on a plane having by center the projection of the pole which has oriented them. While the centrosomes are yet little apart from one another, it is problabe, if they have any influence upon the centromeres, that they aid the chromosomes in distending themselves horizontally on that plane. However, it is not obligatory that all the chromosomes take their position of equilibrium at the same time. While some ones have already done this, some others may still be in an oblique or perpendicular position. The speed with which the centrosomes go apart from one another for creating the bipolari- 
ty in the cell may be such, that the chromosomes which have not yet taken an horizontal position perhaps never do it, passing directly from the position they have to the oriented metaphase position. In Euryophthalmus humilis the bipolarity takes some time to be established, reason by which all the chromosomes lie down horizontally. (Fig. 28 and Photo 1). Nevertheless, the fact that some chromosomes can be seen in positions other than the horizontal, may cnly signify that they had not yet taken that position in the moment the cell was fixed. The fact is that the aspects which precede the bipolarity and therefore the orientation, simulate a metaphase in which some chromosomes are lying while others are standing with regard to the plane they occupy.

In the Hemiptera studied elsewhere by the present writer many primary metaphases have been found in which some chromosomes instead of being in the expected position, that is, with the ends turned towards the poles, were lying on the equatorial plane. Such a fact, contrarily to what HELENIUS (1952) thinks, does not mean two different modes of orientation, auto-orientation and co-orientation respectively. The great duration of the primary metaphase stage is undoubtedly correlated with the slowness with which the chromosomes orient. The chromosomes disposed more or less parallelly to the equador of the spindle are justly those which have not yet been oriented. it is possible that they get orientation only when the forces operating in the anaphase begin to be active. In this occasion one can easily verify that all the tetrads are stretched parallelly to the spindle axis. Auto-orientation, therefore, means only that the tetrads are not yet under the orienting influence of the poles.

NORONHA-WAGNER \& DUARTE DE CASTRO (1952b) studying the meiosis of Luzula nemorosa have found that the behaviour of the chromosomes of that plant in both divisions is exactly that described by PIZA in Hemiptera. However, scaping the evidence of the facts, the authors, for explainig the phenomenon, have conceived a scheme based on the supposition that the chromosomes were provided with diffuse centromere, which I repute entirely unadequate. In fact, if all things point out, as recognized by the authors, that the chromosomes orient and divide as if the polar actions worked only at the ends, why to make appeal to assumptions devoid of objetive supports ? Really, if the unique indication leading to the idea of 
diffuse attachment are the orientation and the movement of the chromosomes parallelly to the equator, one does not think in such an attachment modus since the chromosomes orient and move parallelly to the spindle axis. Moreover, pulled towards the poles by forces applied to their whole length, no chromosomes should be mechanically able to assume a position perpendicularly to the plane of the equator.

Neither the scheme nor the photos presented suggest that the metaphase or anaphase chromosomes have resulted from a primary pairing of the arms of each chromosome followed by a secondary pairing of the members of each pair. All indicates, on the contrary, that the homologues have paired, as in Hemiptera, by their whole length. The pairing of the arms of isochromosomes should satisfy the pairing forces, and no other forces would exist for appealing to in order to realize another pairing, furthermore, for the homologues might be much distant apart in the nucleus. The idea of pairing of arms followed by pairing of chromosomes led the authors to complicate the events, admitting pairing in the second division too.

The evident dicentricity of the chromosomes of Luzula nemorosa makes possible to simplify the scheme of the authors by accepting, as it happens in the Hemiptera, that, once finished the first division, a fusion of half-chromosomes and not of half-cromatids independently, takes place in the newly fractured extremities. Thus, the chromosomes which reappear in the secondary metaphase show their two chromatids, an appeal to a second pairing of chromatids that have previously separated being needless. The second division once finished, the halfchromatids in way to the poles fuse again by the acentric extremities, regenerating the iso-chromosomes.

Dicentric chromosomes orient and move in two different ways in meiosis: when the two members of each bivalent are not bound by chiasmata and the centromeres repell one another since much early,they orient parallelly to the equator and keep this orientation while moving (Tityus),(Fig. 31); when, on the other hand, the centromeres do not repulse one another because the pairing force has not yet relapsed, (Fig. 32), or because the two mates are bound together by chiasmata, (Fig 33 ), the bivalents orient and the chromosomes move parallelly to the spindle axis, (Hemiptera). Why, this ? Simply because there being no repulsion of the corresponding extremities, they behave as if a single centromere were present in them. In conditions as such, the influence of the poles would, sooner or later, create a system of forces which should compel the whole 
to rotate until it reaches its equilibrium, what would be realized when its axis became parallel to that of the spindle. (Figs. 34 and 35).

Dicentric univalents when in possession of centromeres already divided at metaphase, orient and move parallelly to the equator (Tityus and Hemiptera spermatogonial chromosomes). When the chromosome has undivided but active centromeres, orientation is parallelly to the spindle axis. (Figs. 34 and 35). (Heterochromosome of some Hemiptera in the heterokinetic division). If the centromeres are inactive the chromosome does not orient, remaining indifferently in any place in the cell (Heterochromosome of other Hemiptera in the heterokinetic division).

The univalent of Luzula campestris (NORONHA-WAGNER \& DUARTE DE CASTRO, 1952a) having undivided but active centromeres, orients, as expected, parallelly to the axis of the spindle.

\section{SUMMARY}

Studying the meiosis of two Hemiptera, mamely, Lybindus dichrous (Coreidae) and Euryophthalmus humilis (Pyrrhocoridae), the author has found new proofs in favor of the existence of a centromere at each end of the chromosomes of the insects belonging to that order.

Following the behaviour of a pair of large autosomes of Lybindus, he was able to verify that in the first division of the spermatocytes, the tetrad they form divides transversely by the middle, giving rise to two V-shaped anaphase chromosomes that go to the poles with the vertex pointing forwardly. From the end of the first division till the metaphase of the second one, the centromeres occupying the vertex of the $\mathrm{V}$ go apart from one another, making the chiasmata existing there slip to the opposite extremities, what changes the $\mathrm{V}$ into an $\mathrm{X}$. When the chiasmata reach the acentric ends, the $X$ is again converted into a V. The V of the secondary metaphase, therefore, differs from the $V$ of the primary anaphase, in being inverted that is, in having the centromeres in the extremity of its arms, and no longer in the vertex as in the latter. The opening out of the chromosomes starting at the centric extremities in order to recuperate the dumbbell shape they show in the secondary anaphase, just in the manner postulated by PIZA, is thus demonstrated. 
In Euryophthalmus humilis it was verified once more, that the heterochromosome, in the secondary spermatocytes, orients parallelly to the spindle axis, accompanying with its ends the anaphase plates as they move to the poles.

The author is in disagreement with NORONHA-WAGNER \& DUARTE DE CASTRO 's interpretation of the behaviour of the chromosomes in meiosis of Luzula nemorosa.

\section{LITERATURE}

HELENIUS, O., 1952 - The mode of bivalent orientation in the Hemiptera. Hereditas, 38: 420-424.

NORONHA-WAGNER, M. \& D. de CASTRO, 1952a - Um cromosoma supranumerário em Luzula campestris. Sc. Gen. 4: 149-153.

NORONHA-WAGNER \& D. de CASTRO, 1952b - Interpretação de um comportamento meiótico observado em Luzula. Sc. Gen. 4 : 154-161.

PIZA, S. de Toledo, 1939 - Comportamento dos cromossômios na primeira divisão dos espermatócitos do Tityus bahiensis. Sc. Gen. 1 : 255-261.

PIZA, S. de Toledo, 1941 - Chromosomes with two spindle attachments in the brasilian Scorpion (Tityus bahiensis Perty). J. Hered. 32 : 423-426.

PIZA, S. de Toledo, 1943a - Meiosis in the male of the brasilian Scorpion Tityus bahiensis. Rev. de Agric. 18 : 249-276.

PIZA, S. de Toledo, 1943b - The uselessness of the spindle fibers for moving the chromosomes. Am. Nat. 74: 422-462.

PIZA, S. de Toledo, 1945 - Estudos citológicos em Hemípteros da familia Coreidae. An. Esc. Sup. Agr. "Luiz de Queiroz", 2 : $119-148$.

PIZA, S. de Toledo, 1946a - Comportamento dos cromossômios na meiose de Euryophthalmus rufipennis Laporte (Hemiptera-Pyrrhocoridae). An. Esc. Sup. Agr. "Luiz de Queiroz", $3: 27-54$. 
PIZA, S. de Toledo, 1946b - Notas sôbre a meiose de Pachylis (Hemipt. Coreidae). An. Esc. Sup. Agr. "Luiz de Queiroz", $3: 55-67$.

PIZA, S. de Toledo, 1946c - Nota prévia sôbre a meiose de Corizus (Lyorhyssus) hyalinus (Hemiptera-Corizidae). An. Esc. Sup. Agr. "Luiz de Queiroz", 3: 141-158.

SCHRADER, F., 1935 - Notes on the meiotic behaviour of long chromosomes. Cytol. 6 : 422-430.

\section{EXPLANATION FOR THE FIGURES}

\section{Lybindus dichrous}

Fig. 1-Spermatogonium with 13 chromosomes.

Fig. 2-Somatic cell of the female with 14 chromosomes.

Fig. 3-Spermatogonium with 15 chromosomes, two of which are supernumeraries.

Fig. 4-Polar view of primary metaphase showing at the top the major tetrad bent in the form of thick V.

Fig. 5-One of the aspects of the major tetrad some time before the primary metaphase.

Fig. 6-Another aspect of the same tetrad in a phase corresponding to that of Fig. 5 .

Fig. 7 - The components of the major tetrad in lateral view of primary anaphase.

Figs. 8 and 9-Two polar views of secondary metaphase showing the V-shaped major chromosome with the opening turned inwardly.

Fig. 10-Two aspects of the major chromosome in side view of secondary metaphase.

Fig. 11 - Side view of secondary anaphase showing the major chromosomes moving parallelly to the spindle axis.

Fig. 12-Polar view of primary metaphase with two supernumerary chromosomes.

Figs. 13, 14 and 15 - Polar views of secondary metaphase with 7, 8 and 9 chromosomes respectively.

Figs. 16-19, Interpretation of the structure and behaviour of the major chromosomes in the first division: 16, univalent with one centromere at each end; 17 and 18, two aspects shown by the major tetrad in a lateral view of the primary metaphase; 19 side view of the components of the major tetrad in the primary anaphase. 
Figs. 20-22, Interpretation of the behaviour of the major chromosomes in the secondary division : 20 and 21, two aspects of the metaphase in side view; 22, side view of the anaphase.

\section{Euryophthalmus humilis}

Fig. 23 - Spermotogonium with 13 chromosomes.

Fig. 24-Polar view of primary metaphase showing at the top the heterochromosome.

Fig. 25 - Side view of primary metaphase showing at the right the heterochromosome.

Fig. 26-Polar view of secondary metaphase showing in the center the heterochromosome.

Fig. 27 - Side view of secondary anaphase. showing the heterochromosome stretched between the two plates.

Fig. 28 - Secondary spermatocyte chromosomes before orientation, lying down in a plane.

Fig. 29-Diagram showing how can the iso-chromosomes pair in order to originate dumbbell-shaped or cross-shaped tetrads.

Fig. 30-Aspects shown by the major chromosomes of Lybindus from the end of the first division till metaphase of the second one (top), and their interpretation (botton).

Figs. 31-35, Explanation for the manner in which tetrads formed by dicentric chromosomes orient. (See the text).

\section{EXPLANATION FOR THE PHOTOGRAPHIES}

1 - Position assumed by the chromosomes before the secondary metaphase.

2 - Two side views of secondary anaphase showing the heterochromosome stretched between the two plates.

3 -Secondary metaphase with the heterochromosome in the center (top), and secondary anaphase with the heterochromosome stretched between the plates (botton).

4 - Secondary anaphase with the heterochromosome reaching one of the poles (center).

(Magnif. circa X 1000). 